\title{
Composição florística de epífitos vasculares no estreito de Augusto César, Floresta Estacional Decidual do Rio Uruguai, RS, Brasil
}

\author{
JULIANA M. ROGALSKI ${ }^{1}$ e ELISABETE M. ZANIN ${ }^{1,2}$
}

(recebido: 26 de dezembro de 2002; aceito: 2 de outubro de 2003)

\begin{abstract}
Floristic composition of the vascular epiphytes of "estreito de Augusto César", Brazilian Semi-Evergreen Forest of Uruguai River, RS, Brazil). The present study was carried out in a Brazilian Semi-Evergreen Forest, exposed to selective logging of tree species, in the "estreito de Augusto César" , RS. The survey of floristic composition of vascular epiphytes was performed in riparian forest. The species registered were classified into categories based on the relationship with the host tree and its position. Seventy species belonging to 30 genera and eight families were registered. The majority of species was classified as characteristic holoepiphyte. The highest number of species was registered on the primary branches.
\end{abstract}

Key words - floristic, vascular epiphytes, vertical stratification

RESUMO - (Composição florística de epífitos vasculares no estreito de Augusto César, Floresta Estacional Decidual do Rio Uruguai, RS, Brasil). O presente estudo foi desenvolvido em Floresta Estacional Decidual do Rio Uruguai, com vegetação alterada por corte seletivo, no estreito Augusto César, RS. O levantamento da composição florística dos epífitos vasculares foi efetuado ao longo da mata ciliar. As espécies registradas foram classificadas em categorias baseadas na relação com o forófito e posição no mesmo. Foram registradas 70 espécies, pertencentes a 30 gêneros e oito famílias. A maioria das espécies foi classificada como holoepífito característico. A maior riqueza de espécies foi registrada nos ramos primários.

Palavras-chave - composição florística, epífitas vasculares, estratificação vertical

\section{Introdução}

Os epífitos constituem cerca de $10 \%$ de todas as espécies vasculares, aproximadamente 25.000 espécies, distribuídas em 84 famílias (Kress 1986) e influenciam positivamente os processos e a manutenção dos ecossistemas (Lugo \& Scatena 1992).

São típicos de florestas tropicais e subtropicais úmidas. No entanto, a abundância e a diversidade são fortemente influenciadas pela mudança de condições ecológicas ao longo de gradientes altitudinais, latitudinais e continentais (Gentry \& Dodson 1987a, b, Gentry 1988). As temperaturas mais ou menos elevadas, associadas às precipitações intensas e regulares podem favorecer o desenvolvimento de comunidades epifíticas altamente diversificadas, cuja composição florística e estrutura comunitária ainda são pouco conhecidas (Waechter 1998). Regionalmente, a distribuição da chuva ao longo do ano parece ser mais importante para o sucesso dos epífitos que o total anual de chuva na área (Gentry \& Dodson 1987a).

1. Universidade Regional Integrada do Alto Uruguai e das Missões, Departamento de Ciências Biológicas, Avenida Sete de Setembro 1621, 99700-000 Erechim, RS, Brasil.

2 . Autor para correspondência: emz@uri.com.br
A estratificação vertical dos epífitos vasculares no interior das florestas, bem como a preferência por região do forófito vem sendo evidenciada, por vários autores, ao longo dos estudos sobre ecologia de epífitos (Johansson 1974, Benzing 1987, Brown 1990, Zimmerman \& Olmsted 1992, Fischer \& Araujo 1995, Waechter 1992, 1998, Ingran \& Nadkarni 1993, Hietz \& Hietz-Seifert 1995, Breier 1999, Schütz-Gatti 2000, Nieder et al. 2000, Bonnet 2001, Kersten \& Silva 2001).

No Brasil, os estudos são recentes e têm sido direcionados ao conhecimento da composição florística e da estrutura das comunidades (Waechter 1992, 1998, Pinto et al. 1995, Fontoura et al. 1997, Breier 1999, Dittrich et al. 1999, Schütz-Gatti 2000, Kersten \& Silva 2001).

Este estudo teve como objetivo registrar as espécies de epífitos vasculares presentes na mata ciliar do estreito de Augusto César, visto que essa área seria encoberta com o reservatório da Usina Hidroelétrica de Itá.

\section{Material e métodos}

Área de estudo - O presente estudo foi desenvolvido, nos anos de 1998 e 1999, ao longo do Estreito de Augusto César, conhecido vulgarmente como estreito do Uruguai, situado no município Marcelino Ramos, RS, fazendo divisa entre os estados do Rio Grande do Sul e Santa Catarina (figura 1). O 
canal com cerca de $5,5 \mathrm{~km}$ de extensão, localizado entre as

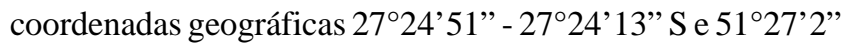
- 5200'03” W (Zanin 1990), e a mata ciliar, foram inundados no ano de 1999, com a instalação da Usina Hidrelétrica de Itá.

A área apresentava vegetação secundária de Floresta Estacional Decidual do Rio Uruguai, por corte seletivo para exploração de madeira. A região apresenta clima, segundo a classificação de Köepen, tipo Cf (temperado chuvoso de ambiente úmido), precipitação anual acima de $1.400 \mathrm{~mm}$ e temperatura média anual de $18^{\circ} \mathrm{C}$ (Eletrosul 1979).

Procedimento - O levantamento de epífitos vasculares foi realizado ao longo do Estreito de Augusto César. Foram realizadas 24 incursões à área, durante os anos de $1998 \mathrm{e}$ 1999, e coletadas todas as espécies de epífitos vasculares registradas. O material coletado foi herborizado, conforme os procedimentos usuais, e tombado no Herbário Balduíno Rambo (HERBARA), Departamento de Ciências Biológicas,

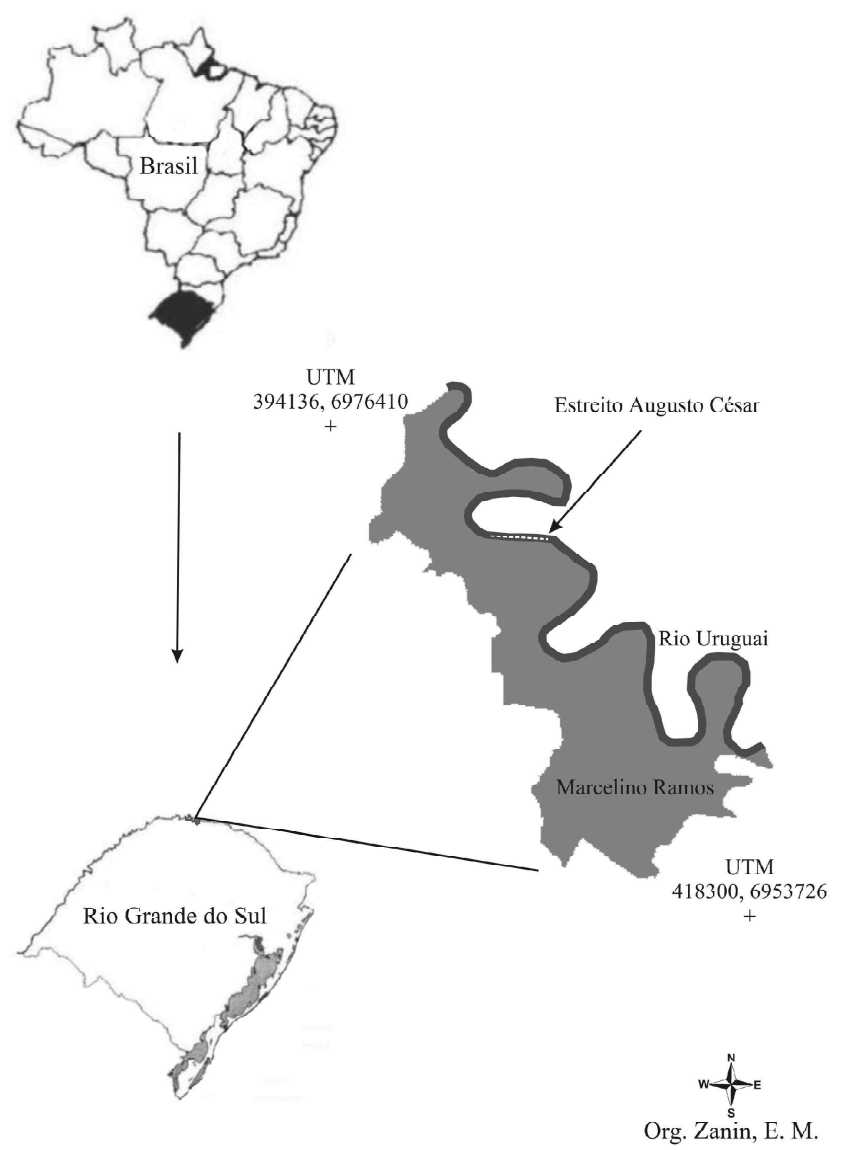

Figura 1. Localização geográfica do estreito de Augusto César e do município de Marcelino Ramos, Estado do Rio Grande do Sul, Brasil.

Figure 1. Geographical localization of "estreito de Augusto César" and Municipality of Marcelino Ramos, Rio Grande do Sul State, Brazil. na Universidade Regional Integrada do Alto Uruguai e das Missões - Campus de Erechim; os números de registro correspondentes encontram-se listados na tabela 1. Os nomes das espécies foram verificadas no Index Kewensis (1997), sendo utilizadas as abreviaturas dos autores sugeridas em Brummitt \& Powell (1992).

As espécies levantadas foram classificadas em categorias ecológicas, conforme sua relação com o forófito (Benzing 1990, 1995) em: holoepífitos característicos (HLC), holoepífitos facultativos (HLF) e holoepífitos acidentais (HLA).

Também foi registrado para cada espécie sua(s) posição(ões) no forófito nas seguintes categorias, adaptadas de ter Steege \& Cornelissen (1989), onde: 1 = metade inferior do tronco, $2=$ metade superior do tronco, $3=$ ramos primários, $4=$ ramos intermediários e $5=$ ramos externos.

\section{Resultados}

Foram registradas 70 espécies, pertencentes a oito famílias e 30 gêneros (tabela 1). As famílias com maior riqueza de espécies foram: Orchidaceae (38 espécies), Bromeliaceae (10 espécies), Piperaceae (oito espécies), Polypodiaceae (seis espécies) e Cactaceae (cinco espécies). Aspleniaceae, Begoniaceae e Commelinaceae apresentaram uma espécie cada. Portanto, a grande maioria de espécies registradas pertence às famílias predominantemente epífitas.

Warmingia eugenii Rchb. f. é uma nova citação para o Estado do Rio Grande do Sul.

Com relação às categorias ecológicas, 54 espécies (77\%) foram classificadas como holoepífitos característicos, 14 espécies (20\%) como holoepífitos facultativos e 2 espécies (3\%) como holoepífitos acidentais (tabela 1).

Com relação à distribuição das espécies nos forófitos, 2 ocorreram na porção inferior do tronco, 25 na porção superior do tronco, 58 ocorreram nos ramos primários, 46 nos ramos intermediários e somente uma nos ramos externos (figura 2). As espécies registradas na parte inferior do tronco (posição 1) são holoepífitos facultativos ou acidentais, na metade superior do tronco e nos ramos intermediários e externos (posições 4 e 5 ) são holoepífitos característicos.

\section{Discussão}

O número de espécies registradas pode ser considerado semelhante ao de outras áreas no sul do País (Waechter 1992, 1998, Dittrich et al. 1996, Breier 1999, Schütz-Gatti 2000, Kersten \& Silva 2001). Porém, alguns estudos têm indicado redução na riqueza e na 
Tabela 1. Categoria ecológica e posição no forófito das espécies de epífitos vasculares, estreito de Augusto César, Floresta Estacional Decidual do Alto Uruguai, RS. HLA = holoepífito acidental; HLF = holoepífito facultativo; HLC = holoepífito característico; $1=$ metade inferior do tronco; $2=$ metade superior do tronco; $3=$ ramos primários; $4=$ ramos intermediários; $5=$ ramos externos.

Table 1. Ecological categories and position of vascular epiphytes on the host tree, "estreito de Augusto César", Brazilian SemiEvergreen Forest, RS. HLA = accidental holoepiphyte; HLF = facultative holoepiphyte; HLC = characteristic holoepiphyte; $1=$ lower middle trunck; $2=$ upper middle trunk; $3=$ primary branches; $4=$ middle branches $; 5=$ outer branches.

\begin{tabular}{|c|c|c|c|}
\hline Família/Espécies & Categoria ecológica & Distribuição vertical & N. registro HER \\
\hline \multicolumn{4}{|l|}{ ASPLENIACEAE } \\
\hline 1. Asplenium divergens Mett. & HLC & 3,4 & 6227 \\
\hline \multicolumn{4}{|l|}{ BEGONIACEAE } \\
\hline 1. Begonia sp. & HLA & 1,2 & 6301 \\
\hline \multicolumn{4}{|l|}{ BROMELIACEAE } \\
\hline 1. Aechmea calyculata Baker & HLF & 2,3 & 6200 \\
\hline 2. Aechmea recurvata (Klotzsch) L.B. Smith & HLF & 3 & 8364 \\
\hline 3. Billbergia nutans Wendl. ex Regel & HLF & 2 & 6012 \\
\hline 4. Billbergia zebrina (Herb.) Lindl. & HLC & 3,4 & 6201 \\
\hline 5. Tillandsia gardneri Lindl. & HLC & 3,4 & 5964 \\
\hline 6. Tillandsia geminiflora Brongn. & HLC & 3,4 & 6202 \\
\hline 7. Tillandsia recurvata $\mathrm{L}$. & HLC & 3,4 & 5961 \\
\hline 8. Tillandsia stricta Sol. & HLC & 2,3 & 6203 \\
\hline 9. Tillandsia tenuifolia $\mathrm{L}$ & HLC & 3,4 & 6204 \\
\hline 10. Vriesea friburgensis $\mathrm{Mez}$ & HLC & 3,4 & 6205 \\
\hline \multicolumn{4}{|l|}{ CACTACEAE } \\
\hline 1. Lepismium cruciforme (Vell.) Miq. & HLC & 3,4 & $6180 ; 6181$ \\
\hline 2. Lepismium houlletianum (Lem.) Barthl. & HLC & 3,4 & 6182 \\
\hline 3. Rhipsalis linearis K. Schum. & HLC & 4 & 5976 \\
\hline 4. Rhipsalis sulcata Web. & HLC & 4 & 5974 \\
\hline 5. Rhipsalis sp. & HLC & 3,4 & 6183 \\
\hline \multicolumn{4}{|l|}{ COMMELINACEAE } \\
\hline 1. Tradescantia sp. & HLA & 3 & 6206 \\
\hline \multicolumn{4}{|l|}{ ORCHIDACEAE } \\
\hline 1. Bulbophyllum regnelli Rchb. f. & HLC & $2,3,4$ & 6151 \\
\hline 2. Campylocentrum aromaticum Barb. Rodr. & HLC & $2,3,4$ & 6191 \\
\hline 3. Campylocentrum ulaei Cogn. & HLC & 3,4 & 6176 \\
\hline 4. Campylocentrum sp. & HLC & 3,4 & 6164 \\
\hline 5. Capanemia micromera Barb. Rodr. & HLC & 4 & 6170 \\
\hline 6. Capanemia superflua (Rchb. f.) Garay & HLC & 3,4 & 6165 \\
\hline 7. Cyclopogon subalpestris Schltr. & HLF & $1,2,3$ & 6017 \\
\hline 8. Cryptophoranthus sp. & HLC & 3 & 6163 \\
\hline 9. Epidendrum densiflorum Hook. & HLC & 3 & 6189 \\
\hline 10. Epidendrum sp. & HLC & 3,4 & 6152 \\
\hline 11. Gomesa planifolia (Lindl.) Kl. ex Rchb. f. & HLC & 4 & 6159 \\
\hline 12. Isochilus linearis (Jacq.) R. Br. & HLC & 3,4 & 6194 \\
\hline 13. Leptotes unicolor Barb. Rodr. & HLC & 3 & 6177 \\
\hline 14. Maxillaria cf. chrysantha Barb. Rodr. & HLC & 3,4 & 6162 \\
\hline 15. Maxillaria picta Hook. & HLC & 3,4 & 6166 \\
\hline 16. Maxillaria porphyrostele Rchb. f. & HLC & 3,4 & 6171 \\
\hline 17. Maxillaria vernicosa Barb. Rodr. & HLC & 4 & 6158 \\
\hline 18. Miltonia flavescens Lindl. & HLC & 3,4 & 6199 \\
\hline 19. Miltonia regnelli $\mathrm{Rchb} . \mathrm{f}$. & HLC & 3,4 & 6144 \\
\hline 20. Oncidium bifolium Sims & HLC & 3,4 & 6041 \\
\hline 21. Oncidium concolor Hook. & HLC & 4 & 6029 \\
\hline
\end{tabular}


continuação

\begin{tabular}{|c|c|c|c|}
\hline Família/Espécies & ia ecológica & Distribuição vertical & N. registro HERBARA \\
\hline 22. Oncidium hians Lindl. & $\mathrm{HLC}$ & $2,3,4$ & 6178 \\
\hline 23. Oncidium pulvinatum Lindl. & HLC & 3,4 & 6190 \\
\hline 24. Oncidium pumilum Lindl. & HLC & 3,4 & 6188 \\
\hline 25. Oncidium cf. widgreni Lindl. & HLC & 3,4 & 6189 \\
\hline 26. Phloeophila similis (Schltr.) Garay & HLC & 2,3 & 6044 \\
\hline 27. Pleurothallis alligatorifera Rchb. f. & HLC & 3 & 6163 \\
\hline 28. Pleurothallis aphthosa Lindl. & HLC & 3,4 & 6169 \\
\hline 29. Pleurothallis crepiniana Cogn. & $\mathrm{HLC}$ & 3 & 6035 \\
\hline 30. Pleurothallis macropoda Barb. Rodr. & HLC & 3,4 & 6025 \\
\hline 31. Pleurothallis obovata Lindl. & HLC & 3 & 6196 \\
\hline 32. Pleurothalis pubescens Lindl. & $\mathrm{HLC}$ & 3,4 & 6195 \\
\hline 33. Pleurothallis sonderana Rchb. $\mathrm{f}$. & HLC & 2,3 & 6147 \\
\hline 34. Pleurothallis sp. 1 & HLC & 3 & 6465 \\
\hline 35. Pleurothallis sp. 2 & $\mathrm{HLC}$ & 3,4 & 6684 \\
\hline 36. Polystachya concreta (Jacq.) Garay \& H.R. Sweet & HLC & $2,3,4$ & 6197 \\
\hline 37. Sophronitis cernua Lindl. & HLC & $2,3,4$ & 6193 \\
\hline 38. Warmingia eugenii Rchb. f. & $\mathrm{HLC}$ & $2,3,4,5$ & 6192 \\
\hline \multicolumn{4}{|l|}{ PIPERACEAE } \\
\hline 1. Peperomia corcovadensis Gardn. & HLF & 23 & 8363 \\
\hline 2. Peperomia delicatula Hench. & $\mathrm{HLF}$ & $2,3,4$ & $6208 ; 6209$ \\
\hline 3. Peperomia martiana Miq. & HLF & 2,3 & $8170 ; 8171$ \\
\hline 4. Peperomia pereskiifolia (Jacq.) H.B.K. & HLF & 2 & 6210 \\
\hline 5. Peperomia rotundifolia Dahlst. & $\mathrm{HLF}$ & 2,3 & 6211 \\
\hline 6. Peperomia tetraphylla (Forst.f.) Hook. \& Arn. & HLF & 2 & 6212 \\
\hline 7. Peperomia trineuroides Dahlst. & HLF & 2 & $6214 ; 6215$ \\
\hline 8. Peperomia urocarpa Fisch. \& Mey. & HLF & 2 & 8169 \\
\hline \multicolumn{4}{|l|}{ POLYPODIACEAE } \\
\hline 1. Campyloneurum nitidum (Kaulf.) C. Presl. & HLF & 3,4 & 6219 \\
\hline 2. Microgramma squamulosa (Kaulf.) Sota & HLC & $2,3,4$ & $6220 ; 6221$ \\
\hline 3. Microgramma vacciniifolia (Langsd. \& Fisch) Copel. & HLC & 2,3 & 8120 \\
\hline 4. Pleopeltis angusta H.B.K. & HLF & $2,3,4$ & $6222 ; 6223$ \\
\hline 5. Polypodium cf. catharinae Langsd. \& Fisch & HLC & $2,3,4$ & 6228 \\
\hline 6. Polypodium squalidum Vell. & HLC & 3,4 & 6225 \\
\hline
\end{tabular}

diversidade de epífitos vasculares em áreas com vegetação alterada (Budowski 1965, Pinto et al. 1995, Barthlot et al. 2001). Assim sendo, o número de espécies registrado neste local também pode ter sido alterado em função de corte seletivo. Portanto, a riqueza de espécies pode ser considerada alta tendo em vista que se trata de uma floresta secundária localizada em uma região subtropical. Isso, provavelmente, se deve à constante umidade local, decorrente da proximidade do rio. Conforme Gentry \& Dodson (1987b), a maior riqueza de epífitos tem sido registrada em florestas úmidas com clima estável.

Dentre as espécies registradas, Miltonia flavenscens Lindl. e $M$. regnelli Rchb. f. encontramse na lista de espécies em risco de extinção do Estado do Rio Grande do Sul (Batista \& Longhi-Wagner 1998).

Com relação à distribuição vertical, a maior riqueza de espécies na base da copa e ramos primários pode ser atribuída, segundo ter Steege \& Cornelissen (1989) e Schütz-Gatti (2000), à maior disponibilidade e qualidade de substrato (bifurcação, ramos horizontais e acúmulo de matéria orgânica) para a fixação de epífitos. Além disso, a grande maioria de espécies registradas é holoepífita característica, categoria que, segundo Benzing (1990), apresenta adaptações relacionadas ao hábito epifítico.

As espécies que ocorrem na parte inferior e superior do tronco são, possivelmente, as que preferem ou toleram ambientes mais úmidos e sombreados, pois requerem condições ambientais semelhantes àquelas 
encontradas no solo, visto que a maioria é holoepífita facultativa ou acidental.

A ocorrência de apenas uma espécie nos ramos externos pode ser atribuída ao fato destes serem muito recentes e dificultarem a instalação de algumas espécies por questões mecânicas e espaciais. Yeaton \& Galdstone (1982) consideraram o tempo que o substrato está disponível como fator determinante na colonização de epífitos.

Schütz-Gatti (2000) também encontrou maior riqueza de espécies nos ramos primários (98 espécies), seguidos dos ramos intermediários (61 espécies), porém a riqueza de espécies encontrada nos ramos externos (61 espécies) foi altíssima quando comparada a este estudo.

A maioria das espécies, entretanto, não foi restrita a uma das posições no(s) forófito(s), o que pode ocorrer em função da variação na altura dos forófitos e da estratificação florestal, pois a distribuição vertical das espécies nos forófitos também é determinada por alguns fatores, tais como a densidade do fluxo de fótons e a umidade (ter Steege \& Cornelissen 1989, Benzing 1995). A luz decresce e a umidade aumenta do dossel até o solo da floresta, formando microclimas diferentes, conforme a altura e a posição no forófito (Parker 1995), os quais são explorados por grupos

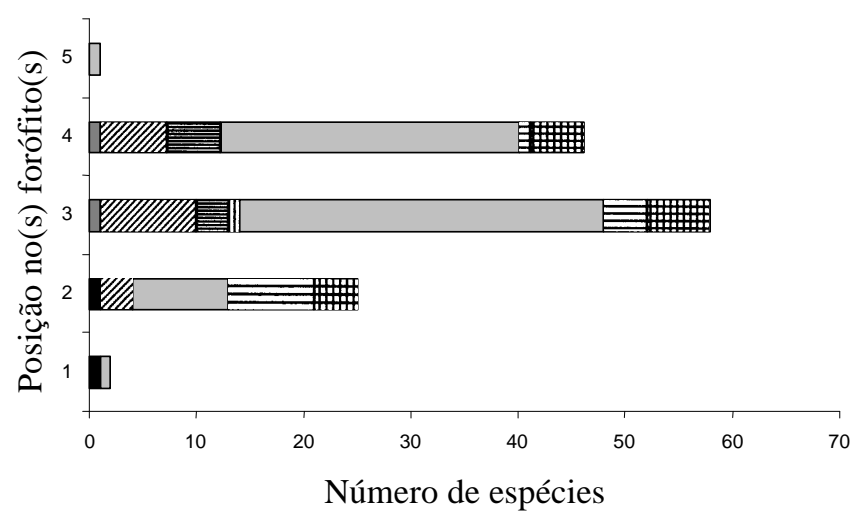

Figura 2. Número de espécies em cada posição do forófito. 1 = metade inferior do tronco; $2=$ metade superior do tronco; 3 = ramos primários; $4=$ ramos intermediários; $5=$ ramos externos. $\bigcirc$ Aspleniaceae; O Begoniaceae; $\mathscr{E}$ Bromeliaceae; DCactaceae; $\square$ Commelinaceae; O Orchidacea; $\equiv$ Piperaceae; Polypodiaceae.

Figure 2. Number of species on each position of the host tree. 1 = lower middle trunck; 2 = upper middle trunk; 3 = primary branches; $4=$ middle branches; 5 = outer branches. - Aspleniaceae; O Begoniaceae; \% Bromeliaceae; Cactaceae; Commelinaceae; O Orchidacea; $\equiv$ Piperaceae; Polypodiaceae. específicos de epífitos. Algumas espécies, porém, ocorreram exclusivamente em determinada posição do forófito.

Alguns autores também constataram preferência por diâmetros de suporte nas copas dos forófitos (Ingran \& Nadkarni 1993), relação entre o tamanho do epífito e o suporte utilizado (Catling \& Lefkovitch 1989) e preferência por forófitos com persistência de casca (Zimmerman \& Olmsted 1992).

Parece haver uma nítida tendência dos holoepífitos acidentais e facultativos ocorrem na metade inferior e na metade superior do tronco, enquanto os holoepífitos característicos ocorrem principalmente nos ramos intermediários dos forófitos. Apesar da variação na altura dos forófitos e de muitas espécies terem ocorrido em mais de uma posição, as espécies podem ser divididas em dois grupos: aquelas que requerem menor luminosidade e maior umidade e aquelas que toleram maior luminosidade e menor umidade. Essa formação de guildas, com condições microclimáticas, é que possibilita a existência da riqueza de epífitos sobre os forófitos.

Agradecimentos - Agradecemos ao Prof. Dr. Jorge Luiz Waechter (UFRGS) pela ajuda na identificação taxonômica das espécies, à Angela Chaves e ao Roberto Aguiar pelo auxílio nas saídas a campo e ao PIBIC/CNPq pela concessão de bolsa à primeira autora deste artigo.

\section{Referências bibliográficas}

BAPTISTA, L.R.M. \& LONGHI-WAGNER, H.M. (coord.) 1998. Lista preliminar de espécies ameaçadas da flora do Rio Grande do Sul. Sociedade Botânica do Brasil, Porto Alegre.

BARTHLOT, W., SCHMIT-NEUERBURG, V., NIEDER, J. \& ENGWALD, S. 2001. Diversity and abundance of vascular epiphytes: a composition of secondary vegetation and primary montane rain forest in the Venezuelan Andes. Plant Ecology 152:145-156.

BENZING, D.H. 1987. Vascular epiphytism: taxonomic participation and adaptive diversity. Annals of the Missouri Botanical Garden 74:183-204.

BENZING, D.H. 1990. Vascular epiphytes. Cambridge University Press, New York.

BENZING, D.H. 1995. The physical mosaic and plant variety in forest canopies. Selbyana 16:159-168.

BONNET, A. 2001. Diversidade e distribuição espacial de bromélias epifíticas em diferentes estádios sucessionais da Floresta Ombrófila Densa, Ilha de Santa Catarina. Dissertação de mestrado, Universidade Federal de Santa Catarina, Florianópolis. 
BREIER, T.B. 1999. Florística e ecologia de epífitos vasculares em uma Floresta Costeira do Sul do Brasil. Dissertação de mestrado, Universidade Federal do Rio Grande do Sul, Porto Alegre.

BROWN, A.D. 1990. EI epifitismo en las selvas montanas del Parque Nacional "EI Rey", Argentina: composición florística y patrón de distribuición. Revista de Biologia Tropical 38:155-166.

BRUMMIT, R.K. \& POWELL, C.E. 1992. Authors of plant names. Royal Botanic Gardens, Kew.

BUDOWSKI, G. 1965. Distribution of tropical american rain forest species in the light of sucessional processes. Turrialba 15:40-42.

CATLING, P.M. \& LEFKOVITCH, L.P. 1989. Associations of vascular epiphytes in a Guatemalan Cloud Forest. Biotropica 21:35-40.

DITTRICH, V.A.O., KOZERA, C. \& SILVA, S.M. 1999. Levantamento florístico de epífitos vasculares no Parque Barigüi, Paraná, Brasil. Iheringia (série Botânica) 52:11-22.

ELETROSUL. 1979. Hidrometeorologia. Bacia Hidrográfica do Rio Uruguai. Estudo de Inventário Hidroenergético. CNEC, v.3, p.1-126.

FISCHER, A.E. \& ARAUJO, A.C. 1995. Spatial organization of a bromeliad community in the Atlantic rainforest, south-eastern Brazil. Journal of Tropical Ecology 11:559-567.

FONTOURA, T., SYLVESTRE, L.S., VAZ, A.M.S. \& VIEIRA, C.M. 1997. Epífitas vasculares, hemiepífitas e hemiparasitas da Reserva Ecológica de Macaé de Cima. In Serra de Macaé de Cima: diversidade florística e conservação da Mata Atlântica (H.C. Lima \& R.R. Guedes-Bruni, eds.). Editora do Jardim Botânico do Rio de Janeiro, Rio de Janeiro, p.89-102.

GENTRY, A.H. \& DODSON, C.H. 1987a. Diversity and biogeography of neotropical vascular epiphytes. Annals of the Missouri Garden 74:205-233.

GENTRY, A.H. \& DODSON, C.H. 1987b. Contribution of nontrees species richness of a tropical rain forest. Biotropica 19:149-156.

GENTRY, A.H. 1988. Changes in plant community diversity and floristic composition on environment and geographical gradients. Annals of the Missouri Garden 75:1-34.

HIETZ, P. \& HIETZ-SEIFERT, U. 1995. Intra and interespecific relations within an epiphyte community in a mexican humid montane forest. Selbyana 16:135-140.

INDEX KEWENSIS. 1997. Oxford University Press, Oxford on compact disc (versão 2.0).

INGRAN, S.W. \& NADKARNI, N.M. 1993. Composition and distribution of epiphytic organic matter in a neotropical cloud forest. Costa Rica. Biotropica 25:370-383.
JOHANSSON, D.R. 1974. Ecology of vascular epiphytes in West African rain forest. Acta Phytogeographica Suecica 59:1-136.

KERSTEN, R.A. \& SILVA, S.M. 2001. Composição florística do componente epifítico vascular em floresta da planície litorânea na Ilha do Mel, Paraná, Brasil. Revista Brasileira de Botânica 24:213-226.

KRESS, W.J. 1986. A symposium: The biology of tropical epiphytes. Selbyana 9:1-22.

LUGO, A.E. \& SCATENA, F.N. 1992. Epiphytes and climate change research in the Caribbean: a proposal. Selbyana 13:123-130.

NIEDER, J, ENGWALD, S., KLAWUN, M. \& BARTHLOTT, W. 2000. Spatial distribution of vascular epiphytes (including hemiepiphytes) in a lowland amazonian rain forest (Surumoni crane plot) of southern Venezuela. Biotropica 32:385-396.

PARKER, G.G. 1995. Structure and microclimate of forest canopies. In Forest canopies (M.D. Lowman \& N.M. Nadkarni, eds.). Academic Press, San Diego, p.73-106.

PINTO, A.C.R., DEMATTÊ, M.E.S.P. \& PAVANI, M.C.M.D. 1995. Composição florística de epífitas (Magnoliophyta) em fragmento de floresta no município de Jaboticabal, SP, Brasil. Científica 23:283-289.

SCHÜTZ-GATTI, A.L. 2000. O componente epifítico vascular na Reserva Natural de Salto Morato, Guaraqueçaba PR. Dissertação de mestrado, Universidade Federal do Paraná, Curitiba.

TERSTEEGE, H. \& CORNELISSEN, J.H.C. 1989. Distribution and Ecology of vascular epiphytes in Lowland Rain Forest of Guyana. Biotropica 21:331-339.

WAECHTER, J.L. 1992. O epifitismo vascular na planície costeira do Rio Grande do Sul. Tese de doutorado, Universidade Federal de São Carlos, São Carlos.

WAECHTER, J.L. 1998. Epifitismo vascular em uma floresta de restinga do Brasil subtropical. Ciência e Natura 20:43-66.

YEATON, R.I. \& GLADSTONE, D.E. 1982. The pattern of colonization of epiphytes on Calabash Trees (Crescentia alata HBK.) in Guanacaste Province, Costa Rica. Biotropica 14:137-140.

ZANIN, E.M. 1990. Flora Espermatófita do Estreito de Augusto César. Marcelino Ramos, RS. Dissertação de mestrado, Universidade Federal do Rio Grande do Sul, Porto Alegre.

ZIMMERMAN, J.K. \& OLMSTED, I.C. 1992. Host tree utilization by vascular epiphytes in a seasonally inundated forest (Tintal) in Mexico. Biotropica 24:402-407. 